\title{
ARTICLE OPEN \\ Controlled mechanical assembly of complex 3D mesostructures and strain sensors by tensile buckling
}

\author{
Xiaogang Guo ${ }^{1}$, Xueju Wang ${ }^{2}$, Dapeng Ou ${ }^{1}$, Jilong Ye ${ }^{2,3,4}$, Wenbo Pang ${ }^{1}$, Yonggang Huang ${ }^{5}$, John A. Rogers ${ }^{6}$ and Yihui Zhang ${ }^{1}$
}

Recent research establishes methods of controlled mechanical assembly as versatile routes to three-dimensional (3D) mesostructures from patterned 2D films, with demonstrated applicability to a broad range of materials (e.g., semiconductors, polymers, metals, and their combinations) and length scales (e.g., from sub-microscale to centimeter scale). Previously reported schemes use pre-stretched elastomeric substrates as assembly platforms to induce compressive buckling of 2D precursor structures, thereby enabling their controlled transformation into 3D architectures. Here, we introduce tensile buckling as a different, complementary strategy that bypasses the need for a pre-stretched platform, thereby simplifying the assembly process and opening routes to additional classes of 3D geometries unobtainable with compressive buckling. A few basic principles in mechanics serve as guidelines for the design of 2D precursor structures that achieve large out-of-plane motions and associated 3D transformations due to tensile buckling. Experimental and computational studies of nearly 20 examples demonstrate the utility of this approach in the assembly of complex 3D mesostructures with characteristic dimensions from micron to millimeter scales. The results also establish the use of nonlinear mechanics modeling as a mechanism for designing systems that yield desired 3D geometries. A strain sensor that offers visible readout and large detectable strain range through a collection of mechanically triggered electrical switches and LEDs serves as an application example.

npj Flexible Electronics (2018)2:14; doi:10.1038/s41528-018-0028-y

\section{INTRODUCTION}

Controlled formation of complex three-dimensional (3D) mesostructures is critically important in various fields of engineering and scientific research, where an increasing emphasis is on promising applications in areas from biomedical devices ${ }^{1-4}$ and energy storage $^{5-7}$ to metamaterial ${ }^{8-10}$ and electronics, ${ }^{11-13}$ and to optical devices. ${ }^{14-17}$ Depending on the length scales and material types, a broad set of manufacturing approaches is now available, including $3 \mathrm{D} / 4 \mathrm{D}$ printing, ${ }^{18-21}$ templated growth, ${ }^{22-24}$ controlled folding, ${ }^{25-}$ 28 and mechanical assembly. ${ }^{29-33}$ Most of these existing approaches are not, however, applicable directly to advanced electronic materials (e.g., single-crystal semiconductors or metals) or to the classes of planar, thin film devices that dominate traditional electronic and optoelectronic systems. For example, in schemes that leverage responsive materials (i.e., gels, ${ }^{34-39}$ shape memory materials, ${ }^{40-43}$ or liquid crystal elastomers, ${ }^{44-46}$ ) integration of inorganic semiconductors, metals, and other electronic materials can be challenging. Approaches that exploit residual stresses $^{12,26,47-50}$ or capillary forces ${ }^{51,52}$ avoid this disadvantage but they involve only relatively simple folding/rolling deformations and access to a limited range of 3D geometries. Mechanically guided schemes that exploit compressive buckling in 3D assembly offer an attractive set of capabilities, including full compatibility with the most advanced planar materials and fabrication methods used in state-of-the-art microsystems technologies. ${ }^{29,30,53}$ Recent reports describe the utility of such approaches in building sophisticated 3D mesostructures in single-crystal silicon, metals, dielectrics, and even in realizing integrated device systems, over length dimensions from sub-micron to centimeter scales. ${ }^{29,30,33,54,55}$ The geometrical transformation process that converts two-dimensional (2D) structures into 3D systems exploits pre-stretched elastomer substrates. Although useful in many contexts, pre-stretching the substrate can cause practical complications in the manufacturing, along with limits in processing options.

Here, we introduce tensile buckling as an alternative approach to the assembly of $3 \mathrm{D}$ mesostructures, with a majority of the demonstrated $3 \mathrm{D}$ topologies inaccessible to compressive buckling. Combined computational and experimental studies of nearly 20 examples show that properly designed 2D precursors can be transformed into desired 3D mesostructures in a deterministic manner, either with use of uniaxial or biaxial tensile strains. A visible strain sensor that relies on mechanically triggered electrical switches and LEDs illustrates a simple 3D device that can be realized using this approach.

\footnotetext{
${ }^{1}$ Department of Engineering Mechanics, Center for Mechanics and Materials and Center for Flexible Electronics Technology, AML, Tsinghua University, 100084 Beijing, People's Republic of China; ${ }^{2}$ Department of Materials Science and Engineering, Northwestern University, Evanston, IL 60208, USA; ${ }^{3}$ State Key Laboratory of Tribology, Tsinghua University, 100084 Beijing People's Republic of China; ${ }^{4}$ Center for Nano and Micro Mechanics, Tsinghua University, 100084 Beijing People's Republic of China; ${ }^{5}$ Departments of Civil and Environmental Engineering, Mechanical Engineering, and Materials Science and Engineering, Northwestern University, Evanston, IL 60208, USA and ${ }^{6}$ Departments of Materials

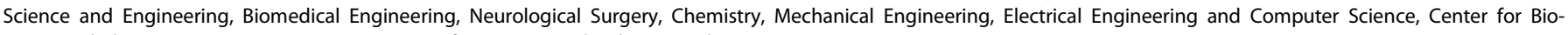
Integrated Electronics, Simpson Querrey Institute for BioNanotechnology, Northwestern University, Evanston, IL 60208, USA

Correspondence: John A. Rogers (jrogers@northwestern.edu) or Yihui Zhang (yihuizhang@tsinghua.edu.cn)

These authors contributed equally: Xiaogang Guo, Xueju Wang and Dapeng Ou.
}

Received: 15 February 2018 Revised: 5 April 2018 Accepted: 9 April 2018

Published online: 14 May 2018 


\section{RESULTS}

Assembly process and design strategy

Figure 1 illustrates the use of tensile buckling for 3D assembly, along with various design strategies in this context. As shown in Fig. $1 \mathrm{a}$, the process begins with transfer printing of $2 \mathrm{D}$ precursors onto an elastomeric substrate (i.e., assembly platform). Strong bonding occurs through surface chemical reactions at lithographically designed sites (i.e., the red regions in Fig. 1a). Stretching (uniaxial in this case) the substrate leads to delamination at the non-bonded regions to induce a 2D-to-3D geometrical transformation through coordinated bending/twisting deformations and translational and rotational motions. See Methods for additional details.

In the tensile buckling approach, the geometry of the 2D precursor is critically important because many 2D layouts either fracture when subjected to large levels of stretching or produce trivial degrees of out-of-plane deformations. Here, we introduce three different design principles. The first leverages the Poisson's effect in which uniaxial tensile stretching of the substrate leads to compression in the transverse direction, thereby triggering 3D assembly through compressive buckling. Figure $1 \mathrm{~b}$ provides four examples of this strategy in bilayers of polyethylene terephthalate (PET; $50 \mu \mathrm{m})$ and copper (Cu; $1 \mu \mathrm{m})$, each by uniaxial stretching along the $y$ axis. Quantitative mechanics modeling based on nonlinear 3D finite element analyses (FEA; see Methods for details) can quantitatively capture all aspects of this assembly process, as evidenced by the remarkable level of agreement between predicted and experimentally realized 3D configurations. The FEA results also show that the maximum principal strains of these 3D mesostructures are well below the fracture thresholds of copper and PET. In the second design approach (Fig. 1c), the bonding sites locate at the inner regions of the 2D precursors, such that the outward movement of those bonding sites induced by stretching of the substrate results in the out-of-plane deformations at the outer regions. Specifically, the closed ribbon circles of the two designs in the right panel of Fig. 1c serve to restrain the in-plane expansion of the precursor structures. The result leads to out-of-plane bending deformations of the ribbons along the radial direction. In this manner, the closed circles experience mainly out-of-plane translations and undergo relative small deformations, thereby minimizing the strain energy of the entire system. The third design applies mainly to spiral-shaped 2D precursors, where uncoiling deformations form 3D mesostructures during tensile buckling. Figure $1 \mathrm{~d}$ summarizes six representative examples based on this principle. The $2 \mathrm{D}$ precursor in the first takes the form of an Archimedean spiral, with bonding sites set at the two ends. The elongation between the two ends induces lateral buckling of the curvy ribbon, and thereby forms an uncoiled 3D ribbon mesostructure. The three designs on the right correspond to variants or combinations of basic Archimedean spirals. It is noteworthy that the 3D geometries demonstrated in Fig. 1c and $d$ are inaccessible to the approaches based on compressive buckling. The aforementioned mesostructures can serve as building block elements to form sophisticated 3D architectures. Figure 1e provides two examples that interconnect the basic elements (i.e., uncoiled 3D ribbon mesostructures in Fig. 1d) in an array configuration. In all cases, the experimental observations agree well with FEA.

These assembly processes involve elastic mechanics and, therefore, they are reversible. The 3D mesostructures recover almost completely to the planar configurations after release of the stretch. This finding is consistent with the FEA, in which the maximum strains of the dominant layer (PET; $50 \mu \mathrm{m})$ are well below the yielding strain. For all of the 3D mesostructures in Fig. 1, the out-of-plane displacements are comparable to the in-plane dimensions, according to their distributions in Supplementary Fig. S1.
Designs optimized to achieve maximum out-of-plane displacements

Achieving large out-of-plane displacements represents an important consideration in precursor design. This section focuses on two representative cases to illustrate the connection between precursor geometries and out-of-plane displacement during the tensile buckling. The first represents the most basic element of a spiral, an arc of angle $\theta$ and end-to-end distance $L$, as shown in Fig. 2a. Here, the arc angle $(\theta)$ represents a dominant geometric parameter that determines the buckled configurations. By contrast, the width-to-length ratio $(W / L)$ typically plays a negligible role. During tensile buckling, the maximum out-of-plane displacement $\left(U_{3, \max }\right)$ of the structure first increases with increasing applied strain ( $\left.\varepsilon_{\text {applied }}\right)$, as shown in Fig. $2 \mathrm{~b}$ and $\mathrm{c}$ for a design with $\theta=240^{\circ}$. As the applied strain reaches $22 \%$, the maximum out-ofplane displacement $\left(U_{3, \max }\right)$ reaches its peak value, beyond which $U_{3, \max }$ decreases, due to the restraint that the finite ribbon length places on the out-of-plane displacement. The deformed configurations in Fig. $2 \mathrm{~b}$ provide further evidence of this behavior. As such, the ratio $U_{3, \max }^{\text {peak }} / L_{\max }$ can serve as a metric for the out-ofplane deformability of a $2 \mathrm{D}$ precursor design during tensile buckling. The experimental and computational result in Fig. $2 d$ show a monotonic increase of $U_{3, \max }^{\text {peak }} / L_{\max }$ with increasing arc angle $(\theta)$. In comparison to the arc angle, the width-to-length ratio $(W / L)$ and the thickness-to-width ratio ( $t / W)$ play relatively minor effects, consistent with experiment and FEA results (see Supplementary Fig. S2a and b for details).

The second design possesses a spiral geometry, consisting of three half-circles with different radii $\left(R_{1}, R_{2}\right.$, and $\left.R_{3}\right)$, as shown in Fig. 2e. In this case, the two radius ratios, $R_{1} / R_{2}$ and $R_{3} / R_{2}$, represent the dominant geometric parameters that affect the buckled configurations. Figure $2 \mathrm{f}, \mathrm{g}$ illustrates the variation of the maximum out-of-plane displacement $\left(U_{3, \max }\right)$ and the deformed configuration as the applied strain increases, for a spiral design with $R_{1} / R_{2}=0.8$ and $R_{3} / R_{2}=1.6$. Here, the out-of-plane displacement also exhibits a non-monotonic dependence on applied strain, indicating the existence of a peak ratio $U_{3, \max }^{\text {peak }} / L_{\max }$ that defines the out-of-plane deformability. Figure $2 \mathrm{~h}$ elucidates the effects of the two radius ratios $\left(R_{1} / R_{2}\right.$ and $\left.R_{3} / R_{2}\right)$ on the out-ofplane deformability. $U_{3, \max }^{\text {peak }} / L_{\max }$ is found to increase monotonically with increasing $R_{1} / R_{2}$ or decreasing $R_{3} / R_{2}$. In comparison to these two ratios $\left(R_{1} / R_{2}\right.$ and $\left.R_{3} / R_{2}\right)$, the width-to-radius ratio $\left(W / R_{2}\right)$ and the thickness-to-width ratio ( $t / W$ ) play relatively minor effects, consistent with experiment and FEA results (see Supplementary Fig. S2c and d for details).

A strain sensor based on mechanically triggered electrical switches and LEDs

The ability to elastically tune the 3D configurations of mesostructures assembled in this manner has important implications in device applications. As an example, we present a visible strain sensor formed by tensile buckling. Compared with the strain sensors reported previously, the 3D strain sensor developed here has the advantages of visible readout and relative large range of detectable strain (from $9.8 \%$ to near $50 \%$ in this example). Figure 3a illustrates the working principle of a basic element, i.e., a mechanically triggered electrical switch connected to a commercial LED. The 2D precursor structure $(\mathrm{Cu} ; 6 \mu \mathrm{m})$ consists of two identical spiral ribbons (each with two half-circles of radii, $R_{1}$ and $R_{2}$ ) separated from one another. Uniaxially stretching the substrate leads to tensile buckling processes that transform the spiral ribbons into 3D configurations. The two separate structures approach one another. As the applied strain reaches a critical value $\left(\varepsilon_{\text {critical }}\right)$, the two ribbons begin to contact, thereby turning the LED on. In general, the critical strain for the onset of contact (and thereby for turning on the LED) depends on the width-toradius ratio $\left(W / R_{1}\right)$ and the radius ratio $\left(R_{2} / R_{1}\right)$. Figure $3 \mathrm{~b}$ 


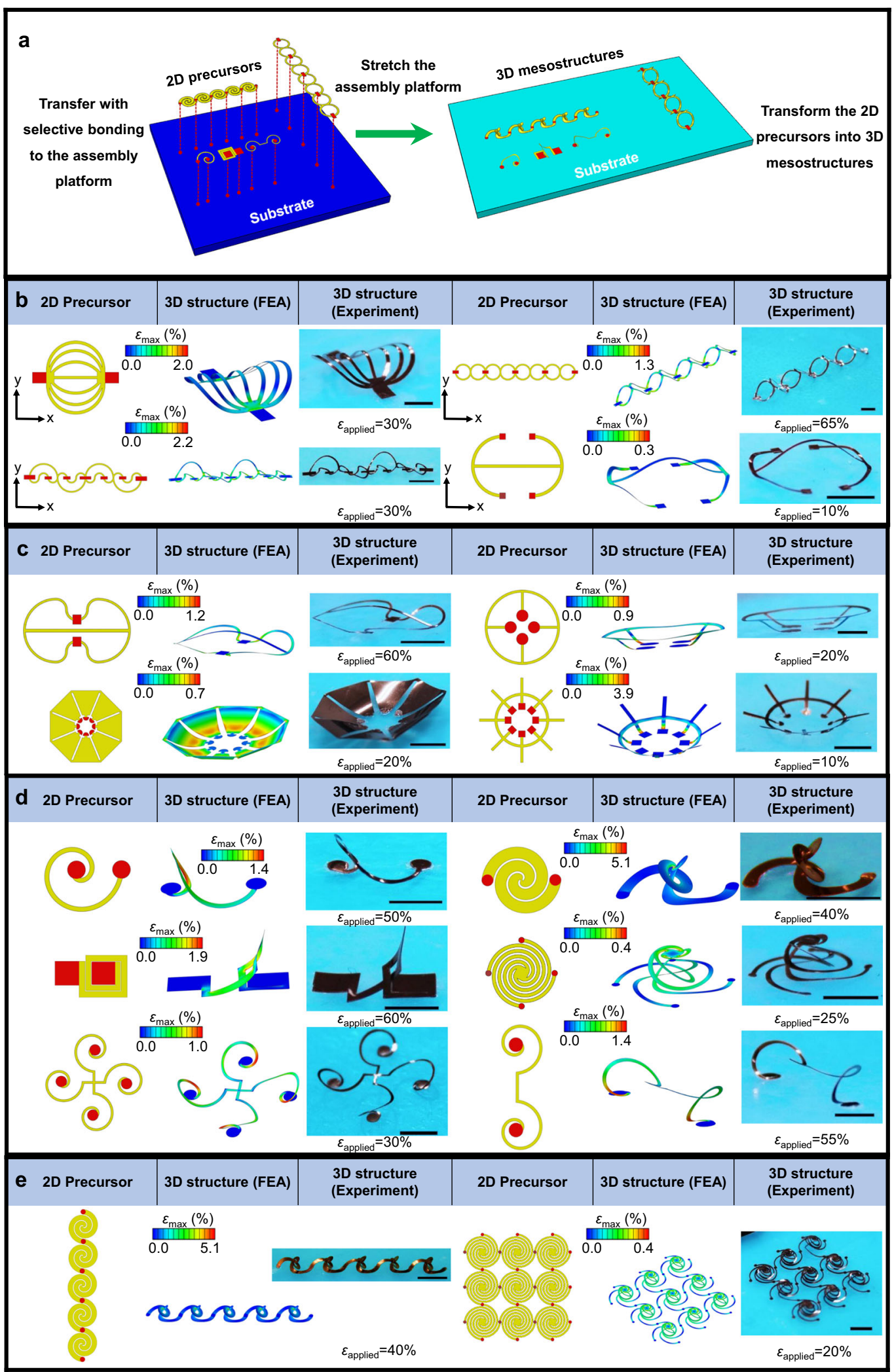

Fig. 1 Design strategies and various examples of 3D mesostructures assembled by tensile buckling. a Results of finite element analyses (FEA) that illustrate the process of assembly by controlled tensile buckling. The red regions of the 2D precursors denote the locations that bond strongly to the substrate. $\mathbf{b} 2 \mathrm{D}$ precursors, experimental images and FEA predictions for four 3D mesostructures in bilayers of PET (50 $\mu \mathrm{m}$ ) and copper $(1 \mu \mathrm{m})$, assembled by $x$-directional compression induced by the Poisson's effect, upon uniaxial stretching of the substrate along the $y$ axis. c Similar results for four 3D mesostructures assembled by lateral buckling under biaxial stretching of the substrate. $\mathbf{d}$ Similar results for another six 3D mesostructures assembled through an uncoiling-type buckling under biaxial stretching of the substrate. e Arrayed architectures with two structures in (d) that serve as the building block elements. The colors in the FEA results correspond to the maximum principal strains. Scale bars, $5 \mathrm{~mm}$ 
a

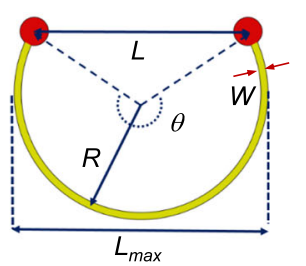

b $\frac{\varepsilon_{\max }(\%)}{0.0}$

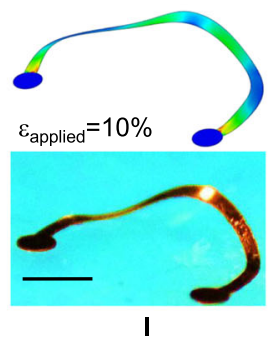

C

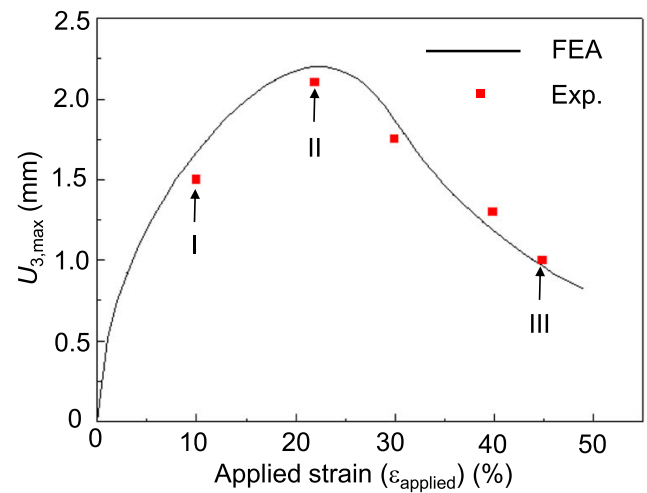

$\varepsilon_{\text {max }}(\%)$

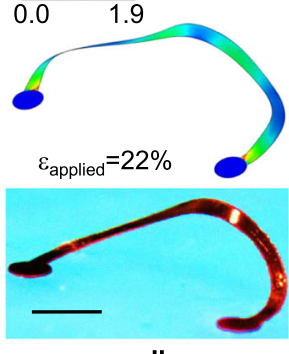

II

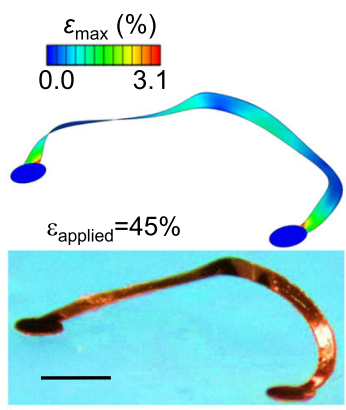

III

d

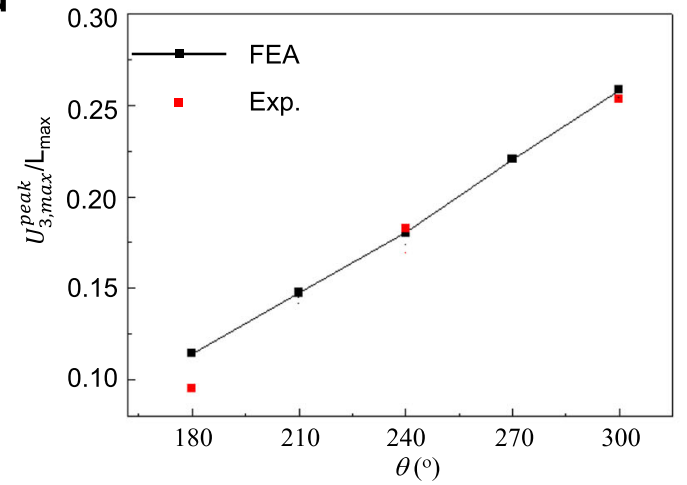

e

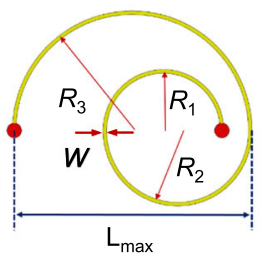

f
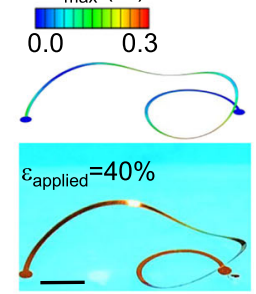

I g

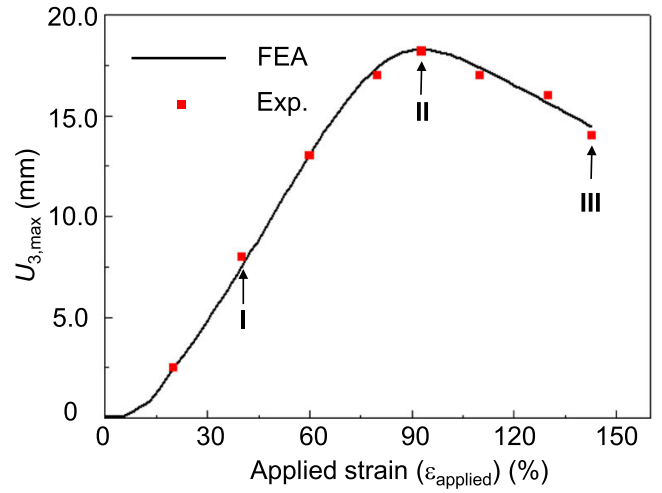

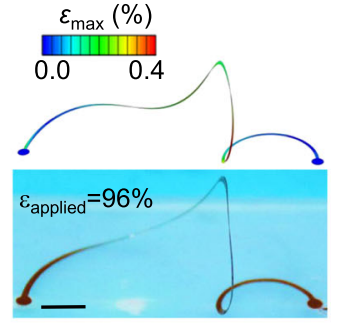

II

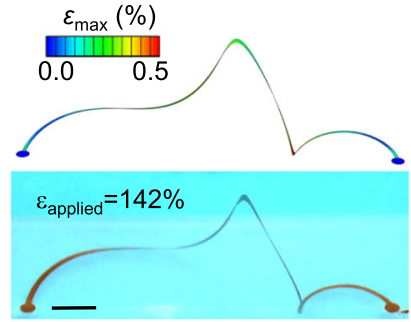

III

h

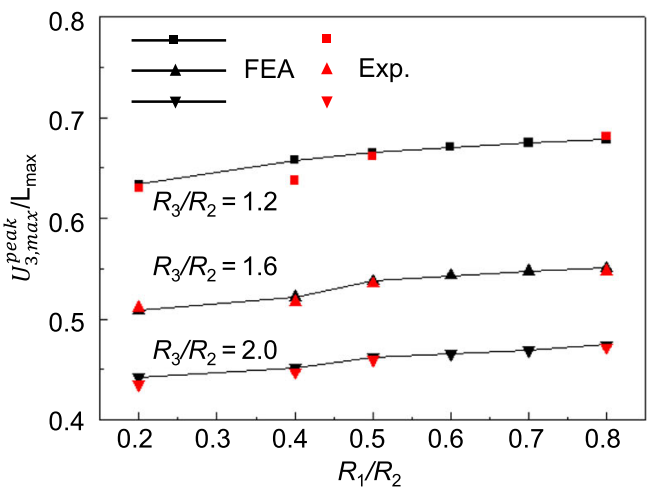

Fig. 2 Designs to optimize the out-of-plane displacements of two representative 2D precursors during tensile buckling. a Illustration of a $2 \mathrm{D}$ precursor in an arc geometry. b Experimental and FEA results on the intermediate states (I, II, and III, as marked in c) of the 3D assembly. c Measured and calculated maximum out-of-plane displacements $\left(U_{3, \max } / L_{\max }\right)$ of the buckled configuration versus the tensile strain applied to the substrate, for the precursor design with parameters $\left(\theta, W / L, t / W, W=\left(240^{\circ}, 0.05,0.1,500 \mu \mathrm{m}\right)\right.$. d Experimental and FEA results on the normalized maximum out-of-plane displacements $\left(U_{3 \max }^{\text {peak }} / L_{\max }\right)$ versus the arc angle $\theta$, for three different ratios $(W / L=0.025,0.033,0.05)$ and fixed parameters $(t / W, W=(0.1,500 \mu \mathrm{m})$. e Illustration of the $2 \mathrm{D}$ precursor in a coil geometry consisting of three half-circles of different radii $\left(R_{1}, R_{2}\right.$ and $\left.R_{3}\right)$. f Experimental and FEA results on the intermediate states (I, II, and III, as marked in $\mathbf{g}$ ) of the 3D assembly. $\mathbf{g}$ Measured and calculated maximum out-of-plane displacements $\left(U_{3, \max }\right)$ of the buckled configuration versus the tensile strain applied to the substrate, for the precursor design with parameters $\left(R_{1} / R_{2}, R_{3} / R_{2}, W / R_{2}, t / W, W\right)=(0.8,1.6,0.05,0.1,500 \mu \mathrm{m})$. $\mathbf{h}$ Experimental and FEA results on the normalized maximum out-of-plane displacements $\left(U_{3, \max }^{\text {peak }} / L_{\text {max }}\right)$ versus the radius ratio $R_{1} / R_{2}$, for three different ratios $\left(R_{3} / R_{2}=1.2,1.6,2.0\right)$ and fixed parameters $\left(W / R_{2}, t / W, W\right)=(0.05,0.1,500 \mu \mathrm{m})$. The colors in the FEA results correspond to the maximum principal strains. The red regions of 2D precursors denote the parts bonded onto the substrate. Scale bars, $5 \mathrm{~mm}$ 
a
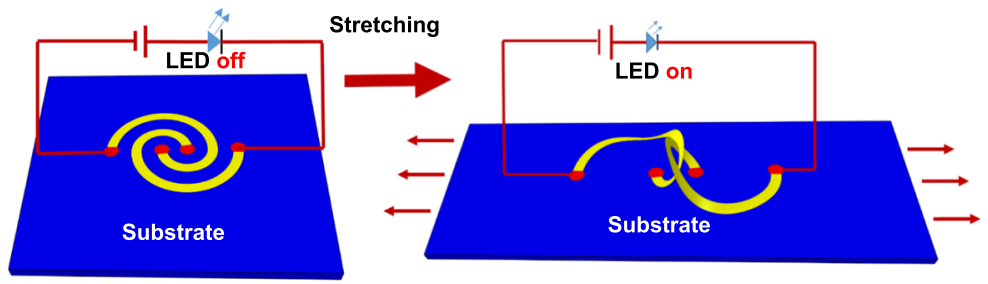

b

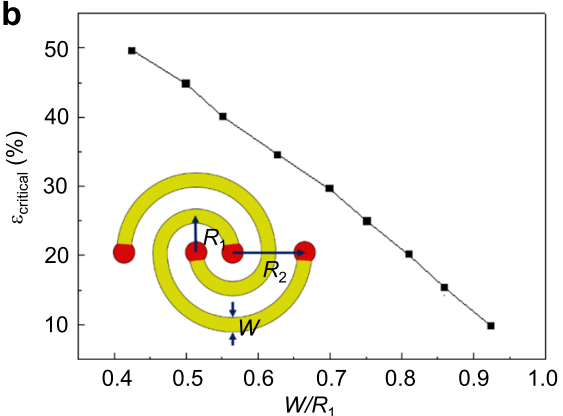

C

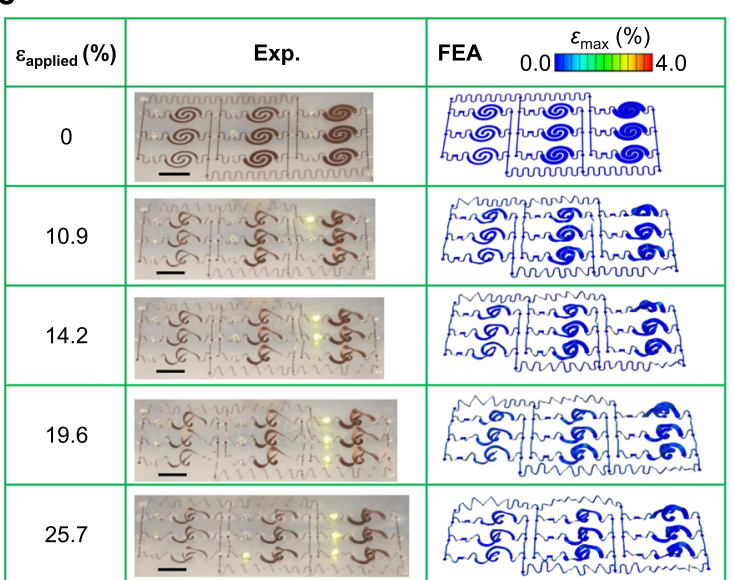

d

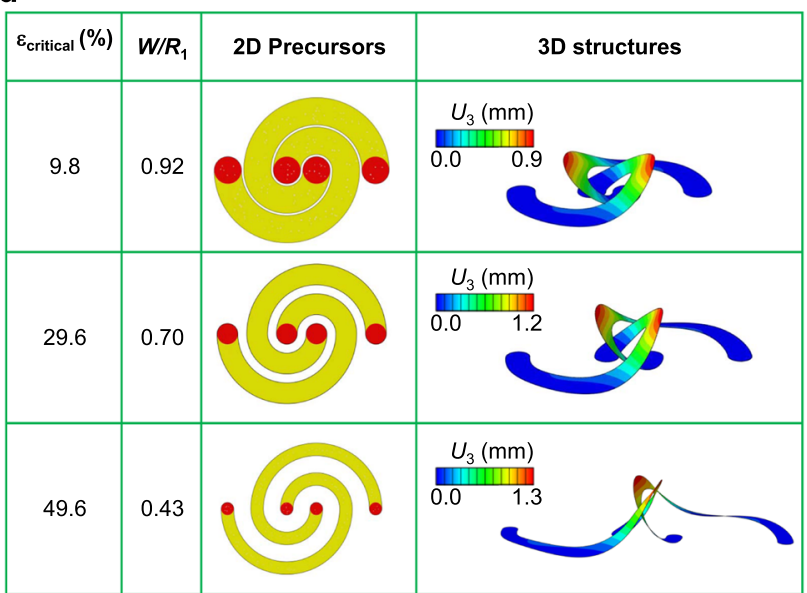

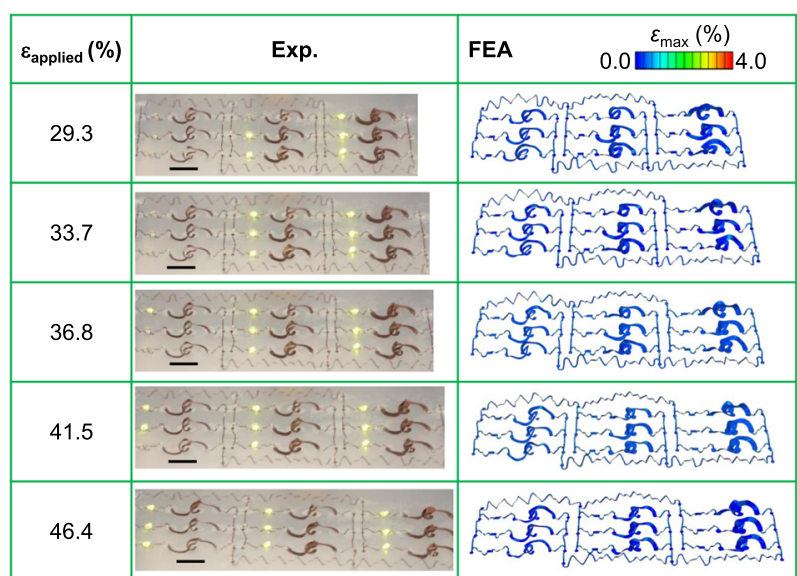

e

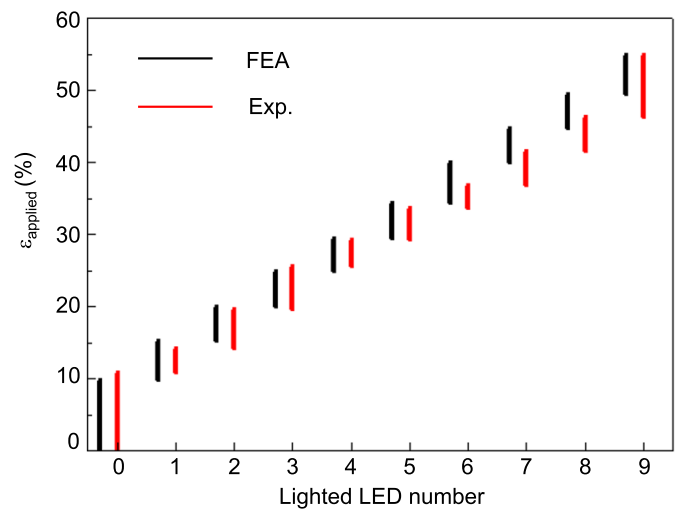

Fig. 3 A 3D strain sensor with LED readout: design principle, quantitative modeling, and experimental measurements. a FEA results that illustrate the design principle of a strain sensor that uses mechanically actuated electrical switches and LEDs. Without stretching, the LED is off; as the tensile strain reaches a critical value, the two electrically conducting ribbons contact each other, thereby turning on the LED. $\mathbf{b}$ FEA predictions of the critical strain for the onset of contact versus the width-to-radius ratio $\left(W / R_{1}\right)$ for a fixed radius ratio $\left(R_{2} / R_{1}=2\right)$. $\mathbf{c}$ Experimental images and FEA predictions on the deformed configurations of a strain sensor at different stages of stretching. The device consists of nine LEDs (lateral dimension: $820 \times 450 \mu \mathrm{m}$ ) and nine electrical-switch elements with different width-to-radius ratios. The colors in the FEA results correspond to the maximum principal strains. d 2D precursor designs and the deformed configurations at the onset of contact (i.e., $\left.\varepsilon_{\text {applied }}=\varepsilon_{\text {critical }}\right)$ for three representative electrical-switch elements. The colors in the FEA results correspond to the out-of-plane displacements. e Measured and computed results of strain ranges under conditions that result in different numbers of lighted LEDs. Scale bars, $4 \mathrm{~mm}$

demonstrates that even for a fixed radius ratio $\left(R_{2} / R_{1}=2\right)$, the critical strain changes dramatically from $\sim 49.6$ to $9.8 \%$, as the width-to-radius ratio $\left(W / R_{1}\right)$ increases from $\sim 0.43$ to 0.92 . This type of mechanical tunability forms the basis of a strain sensor with LED readout by interconnecting spiral-shaped electrical switches with the same radii $\left(R_{1}\right.$ and $\left.R_{2}\right)$ but with different widths $(W)$. Figure $3 c$ and Supplementary Fig. S3a summarize a strain sensor that has nine components connected in parallel. The 2D precursors of three representative components are in Fig. 3d, which offer distinct critical strains $(9.8,29.6$ and $49.6 \%)$ to turn on the LEDs. The 2D precursor designs and detailed FEA results for the other components are provided in Supplementary Fig. S3b. As the stretching of elastomer substrate proceeds, the LEDs are lighted up sequentially as the applied strain $\left(\varepsilon_{\text {applied }}\right)$ reaches the various critical strains of 9.8, 15.3, 20.1, 24.9, 29.6, 34.5, 40, 44.8, and $49.6 \%$. Consequently, the number of activated LEDs serves as 
a visible index of the strain. Full-scale 3D FEA results provide accurate predictions both for the deformed configurations (Fig. $3 c$ ), and for the quantitative values of strain ranges (Fig. 3e) that correspond to different numbers of lighted LEDs. This design concept is scalable to other sizes and geometries, with an example demonstrated in Supplementary Fig. S4, in which the dimensions are scaled up by approximately five times.

\section{DISCUSSION}

In summary, the assembly concepts and design principles reported here provide a route to the rapid formation of complex 3D mesostructures based on tensile buckling. Experimental and computational studies of $\sim 20$ demonstrative examples illustrate several different design strategies and a broad range of 3D geometries. Quantitative mechanics modeling indicates that a maximum in out-of-plane displacement occurs during the buckling process, and that this value can serve as a metric for the realizable level of three dimensionality that can be realized for a given design. A strain sensor with LED readout demonstrates the ability to use theoretical models as design tools to create targeted 3D geometries with desired modes of device functionality. Additional opportunities may follow from the use of concepts with other types of stretchable functional devices.

\section{METHODS}

Finite element analysis

FEA used commercial software (ABAQUS) to capture all mechanics aspects of the 2D-to-3D transformation process, including the distributions of strain during tensile buckling. Four-node shell elements were used for $2 \mathrm{D}$ precursors in PET/copper bi-layer or single layer copper, and eight-node solid elements were used for the elastomeric substrate. Refined meshes ensured computational accuracy. The elastic modulus $(E)$ and Poisson's ratio $(v)$ are $E_{\mathrm{PET}}=3.5 \mathrm{GPa}$ and $v_{\mathrm{PET}}=0.39$ for $\mathrm{PET}, E_{\mathrm{Cu}}=119 \mathrm{GPa}$ and $v_{\mathrm{Cu}}=$ 0.34 for $\mathrm{Cu}$, and $E_{\text {substrate }}=166 \mathrm{kPa}$ and $v_{\text {substrate }}=0.49$ for the substrate.

\section{Fabrication of 3D mesostructures through tensile buckling}

Preparation of 3D mesostructures shown in Figs. 1 and 2 began with mechanical cutting of bilayers of copper $(1 \mu \mathrm{m})$ and PET $(50 \mu \mathrm{m})$ into desired patterns, followed by transfer onto a silicone substrate $(2 \mathrm{~mm}$ in thickness, Dragon Skin ${ }^{\circledast}$, Smooth-On). A commercial adhesive (Super Glue, Gorilla Glue Company), dispensed at desired locations yielded strong mechanical bonds after curing at room temperature for several minutes. Slowly stretching the substrate initiated the assembly process.

\section{Fabrication of the microscale strain sensors with LED readout}

The process began with spin coating (800 r.p.m.) of a precursor to polydimethylsiloxane (PDMS, Sylgard 184, Dow Corning) onto a glass slide. After curing at $70^{\circ} \mathrm{C}$ for $30 \mathrm{~min}, 6-\mu \mathrm{m}$-thick copper foils were laminated onto PDMS-coated glass slides. A thin sacrificial layer of photoresist (AZ4620, about $9 \mu \mathrm{m}$ ) spin cast (3000 r.p.m.) and cured $\left(110^{\circ} \mathrm{C}\right.$ for $1 \mathrm{~min}$ ) on one side of the copper foils facilitated flat and bubble-free contact with the PDMS. Standard photolithography followed by wet etching defined patterns in the copper. Immersing the samples in acetone removed the AZ4620. Water soluble tapes (polyvinyl alcohol, PVA) were used to retrieve the patterned copper foils from the glass slides. With samples on the PVA tapes, a thin layer of silicon dioxide (about $50 \mathrm{~nm}$ ) was deposited at the locations of the bonding sites by electron-beam evaporation through aligned shadow masks. Ultraviolet-ozone treatment of the silicone substrate (Dragon Skin ${ }^{\circledast}$, Smooth-On) and the samples for 4 min followed by lamination and baking $\left(70^{\circ} \mathrm{C}, 10 \mathrm{~min}\right)$ in a convection oven yielded strong bonds at the locations of the silicon dioxide. Rinsing with warm water to dissolve the PVA tape compteted the transfer and bonding process. Nine LEDs (APG0603CGC-TT, Kingbright Company) were bonded to electrode pads with conductive epoxy (CW2400, Chemtronics). The two peripheral pads were connected to conducting wires to allow connection to an external power supply.
Fabrication of the millimeter-scale visible strain sensors Preparation of the millimeter-scale strain sensor in Supplementary Fig. S4 began with mechanical cutting of a film of copper (30 $\mu \mathrm{m}$ in thickness) into desired patterns, followed by transfer to a silicone substrate $(2 \mathrm{~mm}$ in thickness, Dragon Skin ${ }^{\circledast}$, Smooth-On) using thermal releasing tape. A commercial adhesive (Super Glue, Gorilla Glue Company), dispensed at the desired locations on the $2 \mathrm{D}$ precursors, resulted in strong bonding to the silicone substrate after curing at room temperature for several minutes. Commercial LEDs were integrated with the copper patterns using conductive sliver ink. Slowly stretching the substrate initiated the assembly process.

\section{Data availability}

The experimental data which support the findings of this study could be available from the corresponding author upon reasonable request.

\section{ACKNOWLEDGEMENTS}

Y.Z. acknowledges support from the National Natural Science Foundation of China (\#11672152 and \#11722217), the Thousand Young Talents Program of China, and the Tsinghua National Laboratory for Information Science and Technology. Y.H. acknowledges the support from the NSF (\#CMMI1400169, \#CMMI1534120, and \#CMMI1635443)

\section{AUTHOR CONTRIBUTIONS}

X.G., X.W., and D.O. contributed equally to this work. Y.Z. conceived the research; Y.Z. and J.A.R. supervised the research; X.G. and D.O. led the structural designs, mechanics modeling, and millimeter-scale experiments, with assistance from W.P. and Y.H.; X.G. and Y.Z. designed the strain sensor; X.W. and J.Y. experimentally realized the device demonstration of strain sensor at microscale; Y.Z., X.G., and J.A.R. wrote the text and designed the figures. All authors commented on the paper.

\section{ADDITIONAL INFORMATION}

Supplementary information accompanies the paper on the npj Flexible Electronics website (https://doi.org/10.1038/s41528-018-0028-y).

Competing interests: The authors declare no competing interests.

Publisher's note: Springer Nature remains neutral with regard to jurisdictional claims in published maps and institutional affiliations.

\section{REFERENCES}

1. Leong, T. G. et al. Tetherless thermobiochemically actuated microgrippers. Proc. Natl Acad. Sci. USA 106, 703-708 (2009).

2. Tian, B. et al. Macroporous nanowire nanoelectronic scaffolds for synthetic tissues. Nat. Mater. 11, 986-994 (2012).

3. Mannoor, M. S. et al. 3D printed bionic ears. Nano Lett. 13, 2634-2639 (2013).

4. Feiner, R. et al. Engineered hybrid cardiac patches with multifunctional electronics for online monitoring and regulation of tissue function. Nat. Mater. 15, 679-685 (2016).

5. Zhang, H., Yu, X. \& Braun, P. V. Three-dimensional bicontinuous ultrafast-charge and -discharge bulk battery electrodes. Nat. Nanotechnol. 6, 277-281 (2011).

6. Sun, K. et al. 3D printing of interdigitated Li-ion microbattery architectures. Adv. Mater. 25, 4539-4543 (2013).

7. Song, Z. et al. Origami lithium-ion batteries. Nat. Commun. 5, 3140 (2014).

8. Schaedler, T. A. et al. Ultralight metallic microlattices. Science 334, 962-965 (2011).

9. Soukoulis, C. M. \& Wegener, M. Past achievements and future challenges in the development of three-dimensional photonic metamaterials. Nat. Photon. 5, 523-530 (2011).

10. Zheng, $X$. et al. Ultralight, ultrastiff mechanical metamaterials. Science $\mathbf{3 4 4}$, 1373-1377 (2014).

11. Ahn, B. Y. et al. Omnidirectional printing of flexible, stretchable, and spanning silver microelectrodes. Science 323, 1590-1593 (2009).

12. Huang, W. et al. On-chip inductors with self-rolled-up SiNx nanomembrane tubes: a novel design platform for extreme miniaturization. Nano Lett. 12, 6283-6288 (2012).

13. Zarek, M. et al. 3D printing of shape memory polymers for flexible electronic devices. Adv. Mater. 28, 4449-4454 (2016). 
14. Fan, Z. et al. Three-dimensional nanopillar-array photovoltaics on low-cost and flexible substrates. Nat. Mater. 8, 648-653 (2009).

15. $\mathrm{Xu}, \mathrm{H}$. et al. Deformable, programmable, and shape-memorizing micro-optics. Adv. Funct. Mater. 23, 3299-3306 (2013).

16. Lee, J. H. et al. 25th anniversary article: ordered polymer structures for the engineering of photons and phonons. Adv. Mater. 26, 532-569 (2014).

17. Rogers, J., Huang, Y. G., Schmidt, O. G. \& Gracias, D. H. Origami MEMS and NEMS. MRS Bull. 41, 123-129 (2016).

18. Gladman, A. S., Matsumoto, E. A., Nuzzo, R. G., Mahadevan, L. \& Lewis, J. A. Biomimetic 4D printing. Nat. Mater. 15, 413-418 (2016).

19. Wu, J. et al. Multi-shape active composites by 3D printing of digital shape memory polymers. Sci. Rep. 6, 24224 (2016).

20. Zhang, Y. et al. Printing, folding and assembly methods for forming 3D mesostructures in advanced materials. Nat. Rev. Mater. 2, 17019 (2017).

21. Ding, Z. et al. Direct 4D printing via active composite materials. Sci. Adv. 3, e1602890 (2017).

22. Lin, S. Y. et al. A three-dimensional photonic crystaloperating at infrared wavelengths. Nature 394, 251-253 (1998).

23. Noda, S., Tomoda, K., Yamamoto, N. \& Chutinan, A. Full three-dimensional photonic bandgap crystals at near-infrared wavelengths. Science 289, 604-606 (2000).

24. Qi, M. et al. A three-dimensional optical photonic crystal with designed point defects. Nature 429, 538-542 (2004).

25. $\mathrm{Li}, \mathrm{X}$. Strain induced semiconductor nanotubes: from formation process to device applications. J. Phys. D Appl. Phys. 41, 193001 (2008).

26. Cho, J. H. et al. Nanoscale origami for 3D optics. Small 7, 1943-1948 (2011).

27. Li, X. Self-rolled-up microtube ring resonators: a review of geometrical and resonant properties. Adv. Opt. Photon. 3, 366-387 (2011).

28. Pandeya, S. et al. Algorithmic design of self-folding polyhedra. Proc. Natl Acad. Sci. USA 108, 19885-19890 (2013).

29. $\mathrm{Xu}, \mathrm{S}$. et al. Assemble of micro/nanomaterials into complex, three-dimensional architectures by compressive buckling. Science 347, 154-159 (2015).

30. Zhang, Y. et al. A mechanically driven form of kirigami as a route to $3 D$ mesostructures in micro/nanomembranes. Proc. Natl Acad. Sci. USA 112, 11757-11764 (2015).

31. Yan, Z. et al. Controlled mechanical buckling for origami-inspired construction of 3D microstructures in advanced materials. Adv. Funct. Mater. 26, 2629-2639 (2016)

32. Rafsanjani, A. \& Bertoldi, K. Buckling-induced kirigami. Phys. Rev. Lett. 118, 084301 (2017).

33. Yan, Z. et al. Three-dimensional mesostructures as high-temperature growth templates, electronic cellular scaffolds, and self-propelled microrobots. Proc. Nat Acad. Sci. USA 114, E9455-E9464 (2017).

34. Klein, Y., Efrati, E. \& Sharon, E. Shaping of elastic sheets by prescription of nonEuclidean metrics. Science 315, 1116-1120 (2007).

35. Kim, J., Hanna, J. A., Byun, M., Santangelo, C. D. \& Hayward, R. C. Designing responsive buckled surfaces by halftone gel lithography. Science 335, 1201-1205 (2012).

36. $\mathrm{Yu}, \mathrm{C}$. et al. Electronically programmable, reversible shape change in two- and three-dimensional hydrogel structures. Adv. Mater. 25, 1541-1546 (2013).

37. $\mathrm{Wu}, \mathrm{Z}$. L. et al. Three-dimensional shape transformations of hydrogel sheets induced by small-scale modulation of internal stresses. Nat. Commun. 4, 1586 (2013).

38. Breger, J. C. et al. Self-folding thermo-magnetically responsive soft microgrippers. ACS Appl. Mater. Interfaces 7, 3398-3405 (2015).
39. Cangialosi, A. et al. DNA sequence-directed shape change of photopatterned hydrogels via high-degree swelling. Science 357, 1126-1130 (2017).

40. Hawkes, E. et al. Programmable matter by folding. Proc. Natl Acad. Sci. USA 107 12441-12445 (2010).

41. Ge, Q., Qi, H. J. \& Dunn, M. L. Active materials by four-dimension printing. Appl. Phys. Lett. 103, 131901 (2013).

42. Felton, S., Tolley, M., Demaine, E., Rus, D. \& Wood, R. J. Applied origami. A method for building self-folding machines. Science 345, 644-646 (2014).

43. Janbaz, S., Hedayati, R. \& Zadpoor, A. A. Programming the shape-shifting of flat soft matter: from self-rolling/self-twisting materials to self-folding origami. Mater. Horiz. 3, 536-547 (2016).

44. Ware, T. H., McConney, M. E., Wie, J. J., Tondiglia, V. P. \& White, T. J. Voxelated liquid crystal elastomers. Science 347, 982-984 (2015).

45. White, T. J. \& Broer, D. J. Programmable and adaptive mechanics with liquid crystal polymer networks and elastomers. Nat. Mater. 14, 1087-1098 (2015).

46. Yuan, $C$. et al. 3D printed reversible shape changing soft actuators assisted by liquid crystal elastomers. Soft Matter 13, 5558-5568 (2017).

47. Schmidt, O. G. \& Eberl, K. Thin solid films roll up into nanotubes. Nature 410, 168-168 (2001).

48. $\mathrm{Yu}, \mathrm{M}$. et al. Semiconductor nanomembrance tubes: three dimensional confinement for controlled neurite outgrowth. ACS Nano 5, 2447-2457 (2011).

49. Grimm, D. et al. Rolled-up nanomembranes as compact 3D architectures for field effect transistors and fluidic sensing applications. Nano Lett. 13, 213-218 (2013).

50. $\mathrm{Yu}, \mathrm{X}$. et al. Ultra-small, high-frequency, and substrate-immune microtube inductors transformed from 2D to 3D. Sci. Rep. 5, 9661 (2015).

51. Py, C. et al. Capillary origami: spontaneous wrapping of a droplet with an elastic sheet. Phys. Rev. Lett. 98, 156103 (2007).

52. Legrain, A., Berenschot, J. W., Tas, N. R. \& Abelmann, L. Capillary origami of micromachined micro-objects: Bi-layer conductive hinges. Microelectron. Eng. 140, 60-66 (2015).

53. $\mathrm{Fu}, \mathrm{H}$. et al. Morphable 3D mesostructures and microelectronic devices by multistable buckling mechanics. Nat. Mater. 17, 268-276 (2018).

54. Jang, K. I. et al. Self-assembled three dimensional network designs for soft electronics. Nat. Commun. 8, 15894 (2017).

55. Ning, X. et al. 3D tunable, multiscale, and multistable vibrational micro-platforms assembled by compressive buckling. Adv. Funct. Mater. 27, 1605914 (2017).

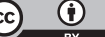

Open Access This article is licensed under a Creative Commons Attribution 4.0 International License, which permits use, sharing, adaptation, distribution and reproduction in any medium or format, as long as you give appropriate credit to the original author(s) and the source, provide a link to the Creative Commons license, and indicate if changes were made. The images or other third party material in this article are included in the article's Creative Commons license, unless indicated otherwise in a credit line to the material. If material is not included in the article's Creative Commons license and your intended use is not permitted by statutory regulation or exceeds the permitted use, you will need to obtain permission directly from the copyright holder. To view a copy of this license, visit http://creativecommons. org/licenses/by/4.0/.

(c) The Author(s) 2018 